EXTENDED REPORT

\title{
Intraocular pressure after replacement of current dual therapy with latanoprost monotherapy in patients with open angle glaucoma
}

\section{E Pillunat, L-I Larsson, and the European and Canadian Latanoprost Study Group*}

*Members listed at the end of paper.

See end of article for authors' affiliations

.....................

Correspondence to: Professor Lutz Pillunat, Universitäts Augenklinik, Fetscherstrasse, D-01307 Dresden, Germany; direction@ uniklinikum-dresden.de

Accepted for publication 30 March 2003
Aims: To evaluate the efficacy and safety of replacing current dual ocular hypotensive therapy with latanoprost $0.005 \%$ monotherapy in patients with open angle glaucoma.

Methods: This randomised, open label, parallel group, multinational study included 466 patients with open angle glaucoma currently on dual ocular hypotensive therapy, including a $\beta$ adrenergic receptor antagonist. Patients were assigned $(1: 3)$ to ongoing dual therapy or a switch to monotherapy with latanoprost $0.005 \%$ once daily for 6 months. Intraocular pressure (IOP) was measured at 10 am and $5 \mathrm{pm}$ at baseline, month 3, and month 6. Groups were compared for differences in diurnal IOP change, IOP success rates (IOP $\leqslant 22 \mathrm{~mm} \mathrm{Hg}$ with $\leqslant 15 \%$ increase from baseline), and clinical success rates (not requiring change in therapy).

Results: Baseline mean diurnal IOP was 17.8 (SD 2.0) $\mathrm{mm} \mathrm{Hg}$ in the latanoprost group and 17.6 (2.1) $\mathrm{mm} \mathrm{Hg}$ in the dual therapy group. After 6 months, mean diurnal IOP was reduced by $0.26(0.18)$ (SEM 1.4\%) $\mathrm{mm} \mathrm{Hg}(p=0.153)$ in the group switched to latanoprost and by $0.37(0.25)(2.1 \%) \mathrm{mm} \mathrm{Hg}$ $(p=0.138)$ in those continuing dual therapy (difference: $0.11 \mathrm{~mm} \mathrm{Hg} ; p=0.641)$. Success rates defined by IOP criteria were $83 \%$ for latanoprost and $89 \%$ for continued dual therapy (difference: $6 \% ; p=0.122$ ). Clinical success rates were $97 \%$ for latanoprost and $99 \%$ for dual therapy (difference: $2 \% ; p=0.161$ ). Ocular adverse events were reported by $23 \%$ of patients in both treatment groups.

Conclusion: Latanoprost monotherapy is a safe and effective alternative for many patients with open angle glaucoma requiring dual topical ocular hypotensive therapy for IOP control.
G laucoma is a leading cause of irreversible blindness worldwide. ${ }^{1}$ Currently, medical treatment for open angle glaucoma is directed at reducing intraocular pressure (IOP) using agents that decrease aqueous humour production or increase aqueous outflow. In many countries, non-selective $\beta$ adrenergic receptor inhibitors, such as timolol, are commonly used as first line therapy. These agents have demonstrated efficacy in lowering IOP and acceptable local tolerability. However, in many patients, IOP may not be sufficiently controlled with topical $\beta$ adrenergic receptor antagonist monotherapy, and additional drugs must be prescribed. ${ }^{23}$

While dual therapy with a $\beta$ adrenergic receptor antagonist plus another topical ocular hypotensive can provide significant further lowering of IOP, ${ }^{45}$ there are several potential drawbacks of such a treatment approach. Increasing regimen complexity may deleteriously affect patient compliance. ${ }^{6-8}$ For example, Gurwitz and colleagues found that elderly glaucoma patients using topical glaucoma medications requiring more than twice daily administration were less likely to refill their prescriptions than their counterparts whose regimens involved less frequent instillation. ${ }^{8}$ In addition, combination therapy involving medications administered at the same time of day can result in reduced efficacy if patients do not wait a sufficient interval between instillation of the different drugs to avoid premature washout of the first medication. Furthermore, the addition of a second agent to the treatment regimen increases ocular exposure to potentially toxic preservatives, such as benzalkonium chloride. ${ }^{10}$

All of the above considerations support a treatment strategy aimed at maintaining monotherapy in glaucoma patients and particularly with a drug requiring only once daily administration. The prostaglandin analogue latanoprost, administered as a single drop in the evening, has demonstrated potent IOP lowering activity in clinical trials of patients with primary open angle glaucoma and ocular hypertension. ${ }^{11-14}$ In three of four studies involving timolol maleate $0.5 \%$ twice daily as an active control, latanoprost $0.005 \%$ once daily was associated with a significantly greater mean diurnal IOP reduction compared with the $\beta$ adrenergic receptor antagonist. ${ }^{11}{ }^{12}{ }^{14}$ Those results suggest that latanoprost monotherapy may offer a viable alternative to dual therapy for patients whose IOP is not sufficiently controlled on a $\beta$ adrenergic receptor antagonist alone.

The purpose of this study was to investigate the role of latanoprost as a replacement for dual ocular hypotensive therapy. Patients with well controlled glaucoma receiving a dual antiglaucoma regimen were randomly assigned to continue existing medication or switch to latanoprost monotherapy for a 6 month period. Intraocular pressures and ocular and systemic safety variables were monitored throughout the study.

\section{MATERIALS AND METHODS}

The study was designed as a randomised, open label, parallel group comparison of current dual therapy, including a $\beta$ adrenergic receptor antagonist, and replacement with latanoprost monotherapy. Eye clinics in 48 locations throughout Belgium, Canada, Germany, Greece, the Netherlands, Spain, Sweden, and the United Kingdom participated. Approval from the appropriate regulatory authorities and ethics committees was obtained for each centre, and all patients provided signed informed consent before study entry. The investigation was conducted according to the guidelines of the Declaration of Helsinki. 
Patients with mild to moderate unilateral or bilateral primary open angle glaucoma, pigmentary glaucoma, or capsular glaucoma were eligible for participation if they were aged 18 years and older and had an IOP $\leqslant 21 \mathrm{~mm} \mathrm{Hg}$ on current therapy consisting of a $\beta$ adrenergic receptor antagonist plus a second topical ocular hypotensive drug. Mild glaucoma was defined as a visual field defect corresponding to a localised paracentral scotoma in the upper or lower half of the visual field. Moderate glaucoma was defined as a visual field defect with a breakthrough to the periphery, but still localised only to the upper or the lower half of the visual field, corresponding to a mean deviation of $<10 \mathrm{~dB}$ in automated perimetry. Visual field testing was done using automated perimetry (threshold program)-for example, Humphrey program 24/2, 30/2, Octopus program Gl, or other threshold perimeter.

Exclusion criteria were previous treatment with latanoprost; a closed or barely open anterior chamber angle; a history of ocular surgery or ocular inflammation within the past 3 months; any history of ocular filtering surgery or severe trauma or argon laser trabeculoplasty; current use of contact lenses or oral ocular hypotensive agents; known hypersensitivity to any ingredients in the study medications; participation in another clinical study within 1 month before the prestudy visit; and any condition preventing reliable applanation tonometry. In addition, women who were pregnant, lactating, or of childbearing potential and not using adequate contraception were excluded from participation.

Eligibility was determined at a prestudy visit that took place within 4 weeks before treatment randomisation (baseline). Evaluations included medical and ocular history, best corrected Snellen visual acuity, refraction, slit lamp examination, ophthalmoscopy through dilated pupils, visual field testing with an automated perimetry threshold program, and IOP measurement (Table 1). Follow up visits were conducted after 4 weeks, 3 months, and 6 months of treatment. The baseline, month 3 and month 6 visits were full day visits. Study procedures repeated at each follow up visit included determination of best corrected Snellen visual acuity, slit lamp examination of the anterior chamber with assessment of cells and flare, review of symptomatology, and direct observation and collection of reports of adverse events. Visual field testing and ophthalmoscopy were also performed at the final study visit.

At baseline, patients were randomised 1:3 to continue their current dual therapy or to switch to latanoprost $0.005 \%$ once daily administered in the evening at $10 \mathrm{pm}$. (Sample size calculations are described in the Statistical methods section). Patients in the dual therapy group were asked to instil morning drops at approximately 8 am and instructed to wait 10-15 minutes between drops when using two medications at the same time. Instructions were also given to instil morning medications at least 2 hours before $10 \mathrm{am}$ on the day of the baseline, month 3, and month 6 visits.

IOP was measured at 10 am and 5 pm during the baseline, 3 and 6 month visits and once only, before $12 \mathrm{pm}$, during the week 4 visit using the same calibrated Goldmann applanation tonometer. IOP was measured three times in each eye, starting with the right eye and alternating between eyes. For each eye, the mean of the three measurements was calculated. In patients requiring bilateral treatment, the values for both eyes were averaged for each assessment time if each eye fulfilled all eligibility criteria. Otherwise, only the eye satisfying the inclusion criteria was included as the study eye, although the fellow eye could be treated with allocated study medication. Safety assessments included all treated eyes. The 10 am and $5 \mathrm{pm}$ data were averaged to determine the diurnal IOP.

\section{STATISTICAL METHODS}

The primary objective of this study was to demonstrate that IOP would be successfully controlled in more than $50 \%$ of patients at 6 months after being switched from an effective dual antiglaucoma regimen to latanoprost monotherapy. Therefore, IOP success was defined as diurnal IOP $\leqslant 22 \mathrm{~mm} \mathrm{Hg}$ with $\mathrm{a} \leqslant 15 \%$ increase from baseline. Clinical success, defined as the investigator's determination that a change in therapy from the baseline assignment was not needed, was analysed as a secondary efficacy endpoint.

Analyses were based on an intent to treat (ITT) population of patients who received at least one drop of study medication and had at least one available IOP value on treatment. For withdrawn patients, the last available IOP measurement was carried forward to month 6. An analysis of covariance was performed with mean diurnal IOP change from baseline to month 6 as response, treatment group and centre as study effects, and baseline diurnal IOP as covariate. Results are reported as least square means (SEM). Treatment groups were compared by constructing a $95 \%$ two sided confidence interval (CI) for the least square estimate of the difference in mean diurnal IOP change (latanoprost minus dual therapy). Per protocol analyses, which excluded patients who did not complete the study or who had major protocol violations, were also conducted; because the ITT and per protocol approaches gave similar results, data from the ITT analyses are presented. All adverse events were recorded regardless of the investigator's assessment of relation to study medication, and the significance of differences between groups was tested using Fisher's exact two sided $\chi^{2}$ test. Analyses were performed with SAS (Version 8.0) (SAS Institute, Cary, NC, USA).

Sample size calculations were based on construction of a two sided 95\% CI for IOP success rates. A sample size of 393 patients in the latanoprost group was calculated assuming a treatment success rate $\geqslant 57 \%$; corresponding calculations for

\begin{tabular}{|c|c|c|c|c|c|c|c|c|}
\hline \multirow[b]{2}{*}{ Examination } & \multirow{2}{*}{$\begin{array}{l}\text { Within } 4 \text { weeks } \\
\text { of baseline }\end{array}$} & \multicolumn{2}{|c|}{ Baseline } & \multirow{2}{*}{$\begin{array}{l}4 \text { Weeks } \\
\text { Before } \\
\text { noon }\end{array}$} & \multicolumn{2}{|c|}{3 Months } & \multicolumn{2}{|c|}{6 Months } \\
\hline & & $10 \mathrm{am}$ & $5 \mathrm{pm}$ & & $10 \mathrm{am}$ & $5 \mathrm{pm}$ & $10 \mathrm{am}$ & $5 \mathrm{pm}$ \\
\hline Medical and ocular history & $x$ & & & & & & & \\
\hline Gonioscopy & $\mathrm{x}$ & & & & & & & \\
\hline Visual field & $\mathrm{x}$ & & & & & & $\mathrm{x}$ & \\
\hline Ophthalmoscopy & $\mathrm{x}$ & & & & & & $\mathrm{x}$ & \\
\hline Symptomatology & $x$ & $\mathrm{x}$ & & $\mathrm{x}$ & $x$ & & $\mathrm{x}$ & \\
\hline Visual acuity & $\mathrm{x}$ & $\mathrm{x}$ & & $\mathrm{x}$ & $\mathrm{x}$ & & $\mathrm{x}$ & \\
\hline Refraction & $\mathrm{x}$ & $\mathrm{x}$ & & & & & & \\
\hline Slit lamp examination & $\mathrm{x}$ & $\mathrm{x}$ & & $\mathrm{x}$ & $\mathrm{x}$ & & $\mathrm{x}$ & \\
\hline Intraocular pressure & $\mathrm{x}$ & $\mathrm{x}$ & $\mathrm{x}$ & $x$ & $\mathrm{x}$ & $\mathrm{x}$ & $\mathrm{x}$ & $\mathrm{x}$ \\
\hline
\end{tabular}


the dual therapy group assumed $\geqslant 80 \%$ treatment success and yielded a sample size of 126 patients. Treatment assignment was determined according to a computer generated randomisation code, stratified by treatment centre, prepared by Pharmacia Corporation Biostatistics and Data Management, Sweden.

\section{RESULTS}

The study included 466 eligible patients, of whom 351 were randomised to latanoprost monotherapy and 115 to continue their existing dual regimen. The baseline characteristics of the two treatment groups are presented in Table 2. Three patients with ocular hypertension were included in the study, all in the latanoprost treatment group. These patients were included in both ITT and per protocol analyses. There were no significant differences between the two treatment groups with respect to age, sex, or diagnosis. All patients were receiving $\beta$ blockers at baseline; second ocular hypotensive baseline medications are summarised in Table 3.

A total of 348 latanoprost treated patients and 114 patients who continued dual therapy were included in the ITT analyses. The randomised patients not included in the ITT population either did not receive any study medication and/or did not have their IOP measured while receiving the study drug. Twenty nine patients (27 latanoprost, two dual therapy) withdrew from the study during the 6 month treatment period. In the latanoprost group, the most common reasons specified were adverse events $(n=12)$ and uncontrolled IOP $(n=5)$. Reasons given by the two withdrawn patients in the dual therapy group were uncontrolled IOP and consent withdrawn.

Figure 1 depicts mean diurnal IOP values during the study and shows IOP remained stable and similar in both groups throughout the 6 months of treatment. Baseline mean diurnal IOP was 17.8 (SD 2.0) $\mathrm{mm} \mathrm{Hg}$ in the latanoprost treatment group and $17.6(2.1) \mathrm{mm} \mathrm{Hg}$ in the continuing dual therapy group. After 6 months of treatment, the mean diurnal IOP was slightly reduced in each group (latanoprost: $-0.26(0.18)(-1.4 \%) \mathrm{mm} \mathrm{Hg}(\mathrm{p}=0.153)$; dual therapy $-0.37(0.25)(-2.1 \%) \mathrm{mm} \mathrm{Hg}(\mathrm{p}=0.138))$. The difference in mean diurnal IOP reduction between the two treatment groups, $0.11 \mathrm{~mm} \mathrm{Hg}$, also was not significant $(\mathrm{p}=0.641,95 \%$ CI: -0.59 to 0.36$)$. Mean IOP levels at each visit and measurement time are summarised in Table 4.

IOP success after 6 months of therapy was achieved by 289/348 patients (83\%; 95\% CI: 79 to 87 ) in the latanoprost group and by 101/114 patients (89\%; $95 \%$ CI: 83 to 94 ) continuing dual therapy. The $6 \%$ between treatment difference

Table 2 Demographic characteristics of patients in the two treatment groups

\begin{tabular}{|c|c|c|}
\hline Characteristics & $\begin{array}{l}\text { Latanoprost } \\
\text { (n=351) }\end{array}$ & $\begin{array}{l}\text { Current dual therapy } \\
(n=115)\end{array}$ \\
\hline \multicolumn{3}{|l|}{ Age (years) } \\
\hline Mean (SD) & $67(11)$ & $67(11)$ \\
\hline Range & $33-93$ & $30-89$ \\
\hline \multicolumn{3}{|l|}{ Sex } \\
\hline Female & 201 & 64 \\
\hline Male & 150 & 51 \\
\hline \multicolumn{3}{|l|}{ Diagnosis } \\
\hline $\begin{array}{l}\text { Primary open angle } \\
\text { glaucoma }\end{array}$ & 315 & 104 \\
\hline Capsular glaucoma & 26 & 9 \\
\hline Ocular hypertension* & 3 & 0 \\
\hline Mixed diagnosis $†$ & 4 & 1 \\
\hline Other & 3 & 1 \\
\hline
\end{tabular}

Table 3 Second ocular hypotensive baseline medications: number (\%) of patients*

\begin{tabular}{llll}
\hline & $\begin{array}{l}\text { Latanoprost } \\
(\mathbf{n}=351)\end{array}$ & & $\begin{array}{l}\text { Current dual } \\
\text { therapy }(\mathbf{n}=115)\end{array}$ \\
\cline { 2 - 2 } Medication & No $(\%)$ & No $(\%)$ \\
\hline Dorzolamide & $145(41.3)$ & & $46(40.0)$ \\
Fixed combination drugt & $83(23.6)$ & & $29(25.2)$ \\
Pilocarpine & $55(15.7)$ & & $19(16.5)$ \\
Brimonidine & $28(8.0)$ & & $9(7.8)$ \\
Clonidine & $25(7.1)$ & & $8(7.0)$ \\
Dipivefrine & $11(3.1)$ & $3(2.6)$ \\
Apraclonidine & $1(<1)$ & $1(<1)$ \\
Acetazolamide $\neq$ & $2(<1)$ & 0 \\
Brinzolamide & $1(<1)$ & 0
\end{tabular}

*Baseline medications in addition to $\beta$ blockers except in those on fixed combination drugs.

†Patients were on fixed combination drugs that included a $\beta$ blocker and dorzolamide or pilocarpine.

‡Both patients were included in intent to treat analyses, and one was included in per protocol analyses.

was not significant ( $\mathrm{p}=0.122 ; 95 \% \mathrm{CI}:-0.13$ to 0.01 ). Clinical success rates were $97 \%$ (95\% CI: 96 to 99 ) for latanoprost patients (339/348) and 99\% (95\% CI: 97 to 100) for patients receiving dual therapy (113/114). The $2 \%$ between treatment difference was not significant $(\mathrm{p}=0.161 ; 95 \% \mathrm{CI}:-0.04$ to 0.01 ).

Adverse event data are summarised in Table 5. Ocular adverse events accounted for the majority of adverse events in both treatment groups but were primarily mild in nature. The incidence of patients reporting at least one ocular adverse event was $23 \%$ in both the latanoprost and dual therapy groups $(\mathrm{p}=1.0)$. With the exception of visual field deterioration, there were also no significant differences between groups in the rates of individual ocular adverse events. Visual field deterioration occurred in seven $(2 \%)$ latanoprost treated eyes and $10(9 \%)$ dual therapy treated eyes $(p=0.002)$. Increased iris pigmentation was reported as an adverse event in three patients in the latanoprost group and no patient in the usual care group; hypertrichosis was reported in three latanoprost treated patients and in one patient receiving dual therapy.

Three serious ocular adverse events occurred during the study, all involving latanoprost treated patients. One patient was diagnosed with anterior uveitis that was judged related to study medication. Recovery occurred with some sequelae after discontinuation of the study drug and conventional medical therapy. Cystoid macular oedema in a second patient was also judged related to latanoprost. Complete recovery was achieved following withdrawal of latanoprost and

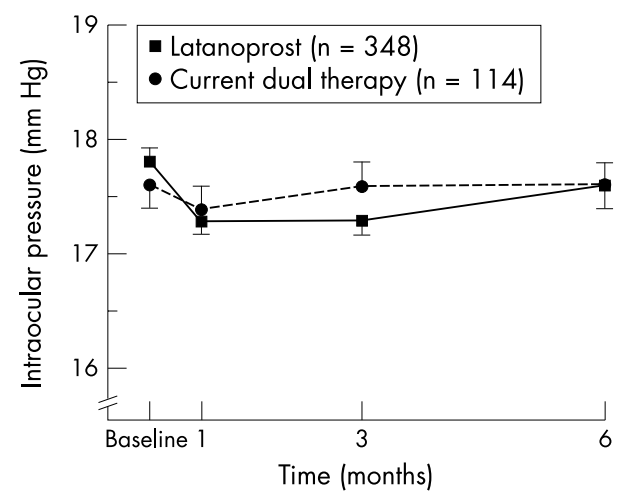

Figure 1 Mean diurnal intraocular pressure (IOP) over the 6 months. Mean (SEM) is given. 
Table 4 Mean intraocular pressure $(\mathrm{mm} \mathrm{Hg})$ over the 6 months at each measurement time (mean (SEM) is given)

\begin{tabular}{lll}
\hline Measurement & Latanoprost $(\mathbf{n = 3 4 8 )}$ & Current dual therapy $(\mathbf{n}=1 \mathbf{1 4})$ \\
\hline Baseline: $10 \mathrm{am}$ & $17.7(0.1)$ & $17.4(0.2)$ \\
$5 \mathrm{pm}$ & $18.0(0.1)$ & $17.8(0.20$ \\
Month 1: $12 \mathrm{noon}$ & $17.3(0.1)$ & $17.4(0.2)$ \\
Month 3: $10 \mathrm{am}$ & $17.3(0.2)$ & $17.5(0.2)$ \\
$5 \mathrm{pm}$ & $17.2(0.1)$ & $17.6(0.2)$ \\
Month 6: $10 \mathrm{am}$ & $17.7(0.2)$ & $17.4(0.3)$ \\
$5 \mathrm{pm}$ & $17.4(0.1)$ & $17.7(0.3)$ \\
\hline
\end{tabular}

appropriate medical therapy. The third patient was diagnosed with a central vein thrombosis that was judged not related to treatment.

Systemic adverse events were reported by a larger proportion of patients in the latanoprost than in the dual therapy group $(13 \% \vee 6 \%$, respectively; $p=0.06)$. Most systemic adverse events were graded as mild and resolved without any sequelae. There were eight serious systemic adverse events, seven in the latanoprost group and one involving a patient receiving dual therapy. None of these events was considered related to study medication.

\section{DISCUSSION}

The results of the present study demonstrate that many patients with glaucoma requiring two topical ocular hypotensive drugs for IOP control can be safely and effectively switched to latanoprost monotherapy. After 6 months, mean diurnal IOP showed no significant change from baseline among patients who substituted latanoprost monotherapy for an existing dual regimen and was comparable to that of the control group who continued combination treatment. In addition, there was no statistical difference between latanoprost and dual therapy when the two treatment groups were compared for IOP and clinical success rates.

Consistent with the findings of this study, other trials have found that latanoprost monotherapy can provide IOP lowering efficacy that is at least comparable to dual therapy. ${ }^{15-17}$ Those studies differed from the current investigation in that they enrolled patients who were being considered for a change in treatment because their IOP was inadequately

Table 5 Ocular and systemic adverse events occurring in $\geqslant 1 \%$ of either treatment group: number of patients in whom the event occurred at least once

\begin{tabular}{lll} 
& $\begin{array}{l}\text { Latanoprost } \\
\text { (n=351) } \\
\text { No (\%) }\end{array}$ & $\begin{array}{l}\text { Current dual therapy } \\
(\mathbf{n}=115) \\
\text { No (\%) }\end{array}$ \\
\hline Ocular adverse event & $19(5.4)$ & $7(6.1)$ \\
Eye irritation & $10(2.8)$ & $5(4.3)$ \\
Cataract/lens opacity & $10(2.8)$ & $4(3.5)$ \\
Conjunctivitis/blepharitis & $8(2.3)$ & $3(2.6)$ \\
Hyperaemia & $7(2.0)$ & $10(8.7)$ \\
Visual field defect & $6(1.7)$ & $4(3.5)$ \\
Vision decreased/blurred & $4(1.1)$ & - \\
Corneal disorder & $1(0.3)$ & $2(1.7)$ \\
Conjunctival disorder & $8(2.3)$ & $2(1.7)$ \\
Systemic adverse event & $7(2.0)$ & - \\
Infection, various & $6(1.7)$ & $3(2.6)$ \\
Arthritis/muscle pain & $5(1.4)$ & - \\
Gastrointestinal symptoms & $4(1.1)$ & - \\
Headache & $3(0.9)$ & $3(2.6)$ \\
Insomnia & $4(1.1)$ & $1(0.9)$ \\
Skin/hair disorder & $3(0.9)$ & - \\
Trauma* & \\
Aggravated hypertension & & \\
\hline *Includes accidents, falls, fractures, etc. & \\
& &
\end{tabular}

controlled on current therapy with one or two topical ocular hypotensive drugs. However, their results showed that compared with timolol run-in, latanoprost monotherapy lowered IOP by $\geqslant 20 \%$, and its efficacy was comparable or superior to add-on therapy with pilocarpine, dorzolamide, or even latanoprost itself.

In this study, IOP was measured at 10 am and $5 \mathrm{pm}$, time points selected to approximate the peak and trough times for the drugs in the dual therapy arm. Recognising that the wide array of drugs administered to patients receiving dual therapy makes precise determination of such times impossible and that latanoprost does not have pronounced peak to trough variation, ${ }^{18}$ the diurnal IOP values obtained in this study can be considered adequate reflections of treatment effects.

In addition to being effective, latanoprost monotherapy offers a safe and well tolerated alternative to dual ocular hypotensive therapy. In this study, the frequency of ocular adverse events was similar in the two treatment groups. Two serious ocular adverse events in latanoprost treated patients were judged to be related to study medication-one case of anterior uveitis and one case of cystoid macular oedema. Inflammation was generally not a problem despite the fact that latanoprost represented newly introduced treatment whereas controls were continuing an established and presumably well tolerated medication regimen. The development of inflammation related adverse events has been a concern with the use of prostaglandin analogues, and cystoid macular oedema, anterior chamber uveitis, and iritis have been reported previously in association with latanoprost use. ${ }^{19}$ However, the rates of such events have been low and the causal relation not established. ${ }^{20}$ Interestingly, the incidence of visual field deterioration was significantly lower among latanoprost treated eyes compared with dual therapy treated eyes. However, that finding should be interpreted carefully given the relatively short duration of the present study and the variability of visual field determinations.

There was a trend for systemic adverse events to occur with a higher frequency in the latanoprost monotherapy group. The higher rate of systemic adverse events in the latanoprost group might be the result of selection bias as the study participants represented patients able to tolerate $\beta$ adrenergic receptor antagonist therapy. Notably, none of the serious systemic adverse events in the latanoprost group were judged related to study medication.

Patient acceptance and medication compliance, factors that can have an important influence on treatment efficacy and disease outcomes, ${ }^{21}{ }^{22}$ were not examined in this study. Compliance with topical ocular hypotensive treatment is notoriously poor..$^{82123}$ While a variety of factors contribute to non-compliance, patient adherence has been consistently found to be inversely related to treatment regimen complexity. ${ }^{721}$ This observation supports the desirability of maintaining long term monotherapy to manage glaucoma and suggests that substituting once daily latanoprost for dual ocular hypotensive treatment may be advantageous. 
In conclusion, latanoprost monotherapy can offer for many glaucoma patients a safe and effective alternative to a dual therapy regimen including a $\beta$ adrenergic receptor antagonist. Switching patients to latanoprost, with its convenient once daily dosing schedule, may result in the additional benefit of enhanced medication compliance.

\section{ACKNOWLEDGEMENT}

Financial support for the study was provided by Pharmacia Corporation.

\section{Authors' affiliations}

L E Pillunat, Department of Ophthalmology, Augenklinik der Techn, Universität Dresden, Germany

L-I Larsson, Department of Ophthalmology, Uppsala University Hospital, Uppsala, Sweden

\section{MEMBERS OF THE EUROPEAN AND CANADIAN LATANOPROST STUDY GROUP}

Ahrens S, MD, Berlin, Germany; Assalian A, MD, Montreal, Canada; Aznares JM, MD, Sevilla, Spain; Bauer W, MD, Starnberg, Germany; Berg K, MD, Eskilstuna, Sweden; Birch M, MD, Newcastle upon Tyne, United Kingdom; Blondeau P, MD, Sherbrooke, Canada; Burger M, MD, Memmingen, Germany; Calissendorff B, MD, Stockholm, Sweden; Christ T, MD, Schorndorf, Germany; Crichton A, MD, Calgary, Canada; Deflorenne, C, MD, Brussels, Belgium; Detry-Morel M, MD, Brussels, Belgium; Deuker H, MD, Darmstadt, Germany; Douglas G, MD, Vancouver, Canada; Fabricius E-M, MD, Berlin, Germany; Fleig H, MD, Würzburg, Germany; Granström P-A, MD, Farsta, Sweden; Hamacher T, MD, Starnberg, Germany; Heist D, MD, Stuttgart, Germany; Hermjacob P, MD, Ahrensburg, Germany; Herzeel R, MD, Brussels, Belgium; Huysmans EG, Alkmaar, Netherlands; Jelinek C, MD, Darmstadt, Germany; Kandarakis A, MD, Athens, Greece; Klein T, MD, Brampton, Canada; Larsson L-I, Uppsala, Sweden; Lesk M, MD, Montreal, Canada; Lindblom B, MD,Gotherburg, Sweden; Marén N, MD, Stockholm, Sweden; Marínez Sánez F, Santander, Spain; Mylopoulos N, MD, Thessaloniki, Greece; Oliva A, MD, Málaga, Spain; Petounis A, MD, Athens, Greece; Pillunat L, MD, Hamburg, Germany; Rademakers AJJM, MD, Nijmegen, Netherlands; Rafat A, MD, Manresa, Spain; Read R, MD, Halifax, Canada; Richter U, Regensburg, Germany; Rietveld E, MD, Amsterdam, Netherlands; Rifkind A, MD, Hamilton, Canada; Roller K-H, MD, Geislinge, Germany; Rosen P, MD, Oxford, United Kingdom; Salcedo J, MD, Bilbao, Spain; Scherzer M-L, MD, Regenstauf, Germany; Schicketanz C, MD, Dinkelsbühl, Germany; Schindler W, MD, Eichstätt, Germany; Schubert T, MD, Memmingen, Germany; Simon Z, MD, Stuttgart, Germany; Söderholm G, MD, Västerås, Sweden; Spencer F, MD, Manchester, United Kingdom; Stilma JS, MD, Utrecht, Nehterlands; Taná P, Alicante, Spain; Taylor J, MD, Victoria, Canada; Ullman U, MD,
Berlin, Germany; Urcelay JL, MD, Madrid, Spain; Waller C, MD, Örebro, Sweden; Webers CAB, MD, Maastricht, Netherlands.

\section{REFERENCES}

1 Thylefors B, Negrel AD, Pararajasegaram R, et al. Global data on blindness. Bull World Health Organ 1995;73:115-21.

2 Uusitalo RJ, Palkama A. Long-term evaluation of timolol. Acta Ophthalmol 1989;67:573-81.

3 Boger WP 3rd, Steinert RF, Puliafito CA, et al. Clinical trial comparing timolol ophthalmic solution to pilocarpine in open-angle glaucoma. Am J Ophthalmol 1978;86:8-18.

4 Hartenbaum D. The efficacy of dorzolamide, a topical carbonic anhydrase inhibitor, in combination with timolol in the treatment of patients with openangle glaucoma and ocular hypertension. Clin Ther 1996;18:460-5.

5 Zimmerman TJ, Canale P. Timolol-further observations. Ophthalmology 1979;86:166-9.

6 Weinreb RN. Compliance with medical treatment in glaucoma. J Glaucoma 1992;1:134-6.

7 Patel SC, Spaeth GL. Compliance in patients prescribed eyedrops for glaucoma. Ophthalmic Surg 1995;26:233-6.

8 Gurwitz JH, Glynn RJ, Monane M, et al. Treatment for glaucoma: adherence by the elderly. Am J Public Health 1993;83:711-6.

9 Chrai SS, Makoid MC, Eriksen SP, et al. Drop size and initial dosing frequency problems of topically applied ophthalmic drugs. J Pharm Sci 1974;63:333-8.

10 Baudouin C. Side effects of antiglaucomatous drugs on the ocular surface. Curr Opin Ophthalmol 1996;7:80-6.

11 Alm A, Stjernschantz J, for the Scandinavian Latanoprost Study Group. Effects on intraocular pressure and side effects of $0.005 \%$ latanoprost applied once daily, evening and morning. A comparison with timolol. Ophthalmology 1995; 102:1743-52.

12 Camras CB, for the United States Latanoprost Study Group. Comparison of latanoprost and timolol in patients with ocular hypertension and glaucoma. A six-month, masked, multicenter trial in the United States. Ophthalmology 1996; 103:138-47.

13 Watson P, Stjernschantz J, for the Latanoprost Study Group. A six-month, randomized, double-masked study comparing latanoprost with timolol in open-angle glaucoma and ocular hypertension. Ophthalmology 1996; 103:126-37.

14 Mishima HK, Masuda K, Kitazawa Y, et al. A comparison of latanoprost and timolol in primary open-angle glaucoma and ocular hypertension. A 12-week study. Arch Ophthalmol 1996;1 14:929-32.

15 Bucci MG, for the Italian Latanoprost Study Group. Intraocular pressurelowering effects of latanoprost monotherapy versus latanoprost or pilocarpine in combination with timolol: a randomized, observer-masked multicenter study in patients with open-angle glaucoma. J Glaucoma 1999;8:24-30.

16 Emmerich K-H. Comparison of latanoprost monotherapy to dorzolamide combined with timolol in patients with glaucoma and ocular hypertension. A 3-month randomised study. Graefes Arch Clin Exp Ophthalmol 2000;238:19-23.

17 Nordmann J-P, Söderström M, Rouland J-F, et al. Comparison of the intraocular pressure lowering effect of latanoprost and a fixed combination of timolol-pilocarpine eye drops in patients insufficiently controlled with betaadrenergic antagonists. Br J Ophthalmol 2000;84:181-5.

18 Larsson L-I. Intraocular pressure over 24 hours after repeated administration of latanoprost $0.005 \%$ and timolol gel-forming solution $0.5 \%$ in patients with ocular hypertension. Ophthalmology 2001; 108: 1439-44.

19 Lindén C, Alm A. Prostaglandin analogues in the treatment of glaucoma. Drugs Aging 1999; 14:387-98.

20 Camras CB. Prostaglandins. In: Ritch R, Shields MB, Krupin T, eds. The glaucomas: glaucoma therapy. 2nd ed. St Louis: Mosby-Year Book, 1996:1449-61.

21 Konstas AG, Maskaleris G, Gratsonidis S, et al. Compliance and viewpoint of glaucoma patients in Greece. Eye 2000;14:752-6.

22 Stewart WC, Chorak RP, Hunt HH, et al. Factors associated with visual loss in patients with advanced glaucomatous changes in the optic nerve head. Am J Ophthalmol 1993;116:176-81.

23 Kass MA, Meltzer DW, Gordon M, et al. Compliance with topical pilocarpine treatment. Am J Ophthalmol 1986;101:515-23. 